\title{
Enhanced Cdk5 Activity and p35 Translocation in the Ventral Striatum of Acute and Chronic Methamphetamine-Treated Rats
}

\author{
Pei-Chun Chen ${ }^{1,2}$ and Jin-Chung Chen ${ }^{* 1,2}$ \\ 'Laboratory of Neuropharmacology, Department of Physiology and Pharmacology, Chang-Gung University, Tao-Yuan, Kwei-Shan, Taiwan, ROC
}

\begin{abstract}
The cyclin-dependent kinase Cdk5 and DARPP-32 (dopamine- and cAMP-regulated phosphoprotein of Mr 32 kDa)-dependent signaling have been implicated in the regulation of dopaminergic neurotransmission after chronic cocaine treatment. In this study, we examined if Cdk5 signaling participates in the behavioral and biochemical effect of acute and chronic methamphetamine (METH) treatment. We found that Cdk5 activity and the membrane fraction of $\mathrm{p} 35$ protein, a Cdk5 activator, in the ventral striatum increased transiently after an injection of $4 \mathrm{mg} / \mathrm{kg} \mathrm{METH}$, while intra-accumbens treatment with a Cdk5 inhibitor, roscovitine, prevented the acute METH-induced locomotor activation. The phosphorylation of DARPP-32 at both Thr75 and Thr34 was differentially regulated after acute METH treatment, but the levels of total Cdk5, p35, and DARPP-32 remained the same. To determine if Cdk5 signaling was associated with behavior sensitization to METH, rats that received repetitive injections of $\mathrm{METH}(4 \mathrm{mg} / \mathrm{kg})$ for 14 consecutive days were analyzed at withdrawal day 7. The results indicate that Cdk5 activity and p35 translocation in the ventral striatum were upregulated in METHsensitized rats; treatment with roscovitine in the nucleus accumbens effectively suppressed the I mg/kg METH-induced behavioral sensitization. Concomitantly, a decrease in the amount of PP-2A and DARPP-32 phosphorylation at Thr34, but an increase in phosphorylation of DARPP-32/Thr75, was observed in the ventral striatum of sensitized rats. The overall results demonstrate that Cdk5/ p35 and downstream signaling in the ventral striatum play a critical role in the effects of acute METH treatment as well as the development of behavioral METH sensitization.
\end{abstract}

Neuropsychopharmacology (2005) 30, 538-549, advance online publication, 10 November 2004; doi: I 0. I 038/s..npp. I 300604

Keywords: methamphetamine; behavioral sensitization; Cdk5; ventral striatum; roscovitine; p35

\section{INTRODUCTION}

Cumulative evidence has revealed that repeated injections of psychostimulants (such as cocaine, amphetamine, and morphine) lead to behavioral sensitization, a phenomenon characterized as a progressive enhancement of the locomotor activity or stereotyped responses (Robinson and Becker, 1986; Kalivas and Stewart, 1991). The mesolimbic dopaminergic projection, from the ventral tegmental area to the nucleus accumbens, plays an essential role during the development of behavioral sensitization (Robinson and Becker, 1986; Kalivas and Stewart, 1991). The early action of psychostimulants in the ventral tegmental area is consid-

*Correspondence: Dr J-C Chen, Department of Physiology and Pharmacology, Chang-Gung University, Room 664, 259 Wen-Hwa I st Road, Tao-Yuan, Kwei-Shan 333, Taiwan, ROC, Tel: + I I| 8863 21।8800ext5282, Fax: + | || 88632118700 ,

E-mail: Jinchen@mail.cgu.edu.tw

${ }^{2}$ Both authors contributed equally to this work.

Received 17 March 2004; revised II August 2004; accepted I5 September 2004

Online publication: 5 October 2004 at http://www.acnp.org/citations/ Npp 100504040 I28/default.pdf ered to be a critical cellular event for initiation of behavioral sensitization (Kalivas and Stewart, 1991). After repetitive drug exposure, the neural circuitry in the ventral striatum (mainly the nucleus accumbens) is required for behavioral expression (Cador et al, 1995). In the ventral striatum, both dopamine $\mathrm{D}_{1}$ - and $\mathrm{D}_{2}$-like receptors and glutamate NMDA and AMPA receptors located on the medium spiny neurons are the primary targets for the altered neurotransmission upon amphetamine or cocaine challenge (Self and Nestler, 1995; Groenewegen et al, 1999). It is known that in behavior-sensitized animals, $D_{1}$ dopamine receptor supersensitivity occurs in the ventral striatum (Nestler and Aghajanian, 1997). This cellular event could functionally associate with upregulated adenylyl cyclase and protein kinase A (PKA) signal, and thus enhances the phosphorylation of the nuclear protein CREB and the expression of immediate-early genes, such as Fos, Jun, and $\triangle$ FosB (Koob, 1996; Keiz et al, 1999). Since the ultimate changes occur at the level of transcription, the behavioral and biochemical abnormalities that result from chronic psychostimulant exposure are considered to be enduring, and $\mathrm{D}_{1}$ receptormediated signaling is viewed as determining predominantly behavioral sensitization. 
Recently, another $D_{1}$ receptor-mediated signaling pathway has been identified in the cocaine-sensitized rats. The $\mathrm{D}_{1}$ receptor-evoked PKA activation in the medium spiny neuron was found to phosphorylate DARPP-32 (dopamineand cAMP-regulated phosphoprotein of $\mathrm{Mr} 32 \mathrm{kDa}$ ) at the threonine 34 position, which in turn inhibits protein phosphatase (PP)-1 and affects a series of downstream physiological effectors (Greengard et al, 1999; Svenningsson et al, 2004). Alternatively, psychostimulants would activate cyclin-dependent kinase Cdk5, and consequently phosphorylate DARPP-32 at the threonine 75 position (Greengard, 2001; Liu et al, 2001). This phosphorylated form of DARPP32 could inhibit the activity of PKA, and thus decrease the phosphorylation of DARPP-32 at Thr34 (White and Cooper, 2001). Reciprocally, PKA would also activate phosphatase PP-2A to dephosphorylate the DARPP-32 at Thr75, and thus inhibit the Cdk5-DARPP-32/Thr75 signaling (Greengard, 2001; Gupta and Tsai, 2001). This signal pathway of Cdk5 and DARPP-32/Thr75 in the ventral striatum is currently postulated to be an endogenous feedback regulatory route, which counterbalances the sensitized behaviors that result from chronic cocaine-triggered $\mathrm{D}_{1}$ receptor/PKA signaling (Nishi et al, 2000; Bibb et al, 2001).

Cdk5 is a serine/proline cellular kinase, which could phosphorylate numerous downstream proteins (Maccioni et al, 2001); it is now known that nuclear $\Delta$ FosB encodes for a Cdk5 transcriptional regulator (Bibb et al, 2001). The induction of $\Delta$ FosB has recently been demonstrated as an essential nuclear signal that mediates cocaine-induced behavioral sensitization (Nestler, 2001). Whether Cdk5 is also involved in methamphetamine (METH)-induced behavioral sensitization, or drug manipulation on in vivo Cdk5 activity would affect the development of METH sensitization is currently unclear, and hence requires further investigation. Previously, we identified that, in amphetamine-sensitized rats, $\Delta$ FosB expression displayed a temporal and spatial variation upon neuroleptic administration (Hsieh et al, 2002). The result suggests a possible linkage between amphetamine sensitization and Cdk 5 signaling. In this study, we intended to investigate if Cdk5 and p35 participate in the acute and chronic METH-evoked behavioral and biochemical events. We found Cdk5/p35 activity and DARPP-32 phosphorylation were significantly altered in the ventral striatum of acute and chronic METH-treated rats. In addition, blockade of Cdk5 activity by intraaccumbens injection of roscovitine prevented behavioral activation as well as behavioral upregulation in METHsensitized rats. The results indicate that $\mathrm{Cdk} 5 / \mathrm{p} 35$ and its downstream signaling display a distinct mode of action, in contrast to cocaine and morphine (Ferrer-Alcon et al, 2003), underlying the METH-evoked behavioral activation.

\section{MATERIALS AND METHODS}

\section{Animal Environment}

Male Sprague-Dawley rats (National Breeding Center, Taiwan) weighting 200-250 g were housed three per cage under a 12-h light/12-h dark cycle (light on at 0700, light off at 1900$)$ and at constant temperature $\left(25^{\circ} \mathrm{C}\right)$ and humidity in a control room under qualified caretakers in the ChangGung Animal Core for at least 1 week before experimenta- tion. Rats were allowed free access to food and water. All the experimental procedures were performed during the light cycle. The ethical guidelines provided by the Chang-Gung Animal Core were followed throughout the study.

\section{Behavioral Assessments}

The acute and chronic effects of METH on Sprague-Dawley rats were evaluated by measuring drug-evoked locomotor activity and stereotypy. After drug administration, the individual rat was tested in an Animal Activity Cage $\left(30 \times 45 \times 45 \mathrm{~cm}^{3}\right.$; MED Associates Inc., St Albans, VT $)$ constructed of opaque plastic walls and four photocells positioned $1 \mathrm{~cm}$ above the floor and spaced evenly along the longitudinal axis of each chamber. When the animal moved, separate interruptions of photocell beams were detected via an electrical interface by a computer situated in an adjacent room. Every 5-min, locomotor activity and stereotypy were recorded for a total session of $2 \mathrm{~h}$. Photo beam interruptions with a duration of less than $0.5 \mathrm{~s}$ were excluded (Chiang et al, 2003).

\section{Surgery and Intracranial Drug Administration}

Rats were anesthetized with $4 \%$ chloral hydrate and light ketamine $(45 \mathrm{mg} / \mathrm{kg}$, i.p. $)$ and placed in a stereotaxic holder (David Kopf Model 900, Tujunga, CA). Two 24-gauge stainless-steel guide cannulae $(5-10 \mathrm{~mm}$ in length) were bilaterally inserted into the nucleus accumbens with one end connected to a $10-\mu \mathrm{l}$ Hamilton syringe via PE-10 tubing. The coordinates for the nucleus accumbens-shell were anterior-posterior 1.7 from bregma, medial-lateral \pm 1.5 from the midline, dorsal-ventral 6.0 from the skull (Paxinos and Watson, 1996). Roscovitine $(10 \mathrm{mM}$; modified from Bibb et al (2001)) or the vehicle (sterile phosphate-buffered saline: $\mathrm{DMSO}=50: 50$ ) was microinfused into the target area at a flow rate of $0.5 \mu \mathrm{l} / \mathrm{min}$ for 2 min driven by a microsyringe pump (CMS 102, Stockholm, Sweden). After completing each injection, the cannula was left in place for an additional 2 min to reduce any possible backflow of the solution along the injection track. The hole was sealed with bone wax immediately afterwards. Animals were removed from the surgical platform and left in their home cage for recovery. After approximately $4 \mathrm{~h}$ after the surgery and when they are fully awake, the rats were tested for METHinduced locomotor activity and stereotypy. METH was administrated intraperitoneally at $1 \mathrm{or} 4 \mathrm{mg} / \mathrm{kg}$ dissolved in sterile $0.9 \%$ saline. The location of the needle tract was histologically verified by Nissl staining after the termination of the experiment.

\section{Tissue Extraction and Western Blot Analysis}

The animals were killed by decapitation, and tissues from the ventral striatum (mostly the nucleus accumbens; Figure 8a) were quickly dissected and immediately frozen in liquid nitrogen. On the day of the experiment, the tissues were either sonicated in $1 \%$ sodium dodecyl sulfate (SDS) buffer and boiled for $5 \mathrm{~min}$, or separated into cytosolic and membrane fractions via CNM compartment protein extraction kit (BioChain Co., Hayward, CA). Small aliquots of the extracts were retained for protein determination by the 
Coomassie blue assay using bovine serum albumin as the standard. Equal amounts of protein $(20-40 \mu \mathrm{g})$ were separated by SDS-polyacrylamide gel electrophoresis (PAGE) (12.5\% polyacrylamide gels), and transferred onto the PVDF membranes (Millipore, Bedford, MA). Membranes were then incubated overnight at $4{ }^{\circ} \mathrm{C}$ with their primary antibody diluted in the TBST (Tris-buffered saline plus $0.1 \%$ Tween-20) buffer. The antibodies used were antityrosine hydroxylase (TH) (1:1000 dilution; Calbiochem, San Diego, CA), anti-Cdk5 (1:500 dilution; Santa Cruz Biotechnology, Santa Cruz, CA), anti-p35 (1:1000 dilution; Santa Cruz Biotechnology), anti-PP-2A catalytic subunit (1:500 dilution; Santa Cruz Biotechnology), anti-phosphoDARPP-32/Thr34 and phospho-DARPP-32/Thr75 (1:1000; general gifts from Drs Greengard/Nairn and from Cell Signaling). After $3 \times 10$-min washes in TBST buffer, the blots were incubated for $1 \mathrm{~h}$ at room temperature with horseradish peroxidase-conjugated secondary antibodies in TBST buffer: 1:2000 goat anti-rabbit IgG (Amersham Pharmacia Biotech, Piscataway, NJ) for Cdk5, p35, pERK1/ 2, phospho-Thr75, and -Thr34 DARPP-32; 1:2000 horse anti-mouse IgG (Amersham Pharmacia Biotech) for TH and DARPP-32; 1 : 4000 goat anti-goat IgG (Amersham Pharmacia Biotech) for PP-2A. Finally, the blots were washed $3 \times 10-\mathrm{min}$ in TBST and developed for $60 \mathrm{~s}$ using the enhanced chemiluminescence (ECL) procedure of Amersham Pharmacia Biotech. The signals were exposed with Hyperfilm (Amersham Pharmacia Biotech) and the blots were stripped and re-probed with anti- $\beta$-actin $(1: 1000$; Sigma Co., St Louis, MO) or anti-DARPP-32 (1:1000 dilution; BD Biosciences, Los Angeles, CA) antibody for quantitative control. The resulting gel bands were quantified by a gel documentation system (Molecular Dynamics) and normalized with $\beta$-actin or DARPP-32 signals.

\section{Assays for Cdk5 Activity}

Cdk5 activity was determined by both radioactivity and SDS-PAGE. Tissues from the ventral striatum were homogenized with a Dounce homogenizer in lysis buffer $(150 \mathrm{mM}$ $\mathrm{NaCl}, 20 \mathrm{mM}$ Tris- $\mathrm{HCl}, 1 \mathrm{mM}$ EDTA, $0.5 \%$ Nonidet P-40, $5 \mathrm{mM}$ dithiothreitol, $1 \mathrm{mM}$ phenylmethylsulfonyl fluoride, $2.5 \mathrm{mM}$ benzamidine, $20 \mu \mathrm{g} / \mathrm{ml}$ antipain, $20 \mu \mathrm{g} / \mathrm{ml}$ leupeptin, $5 \mu \mathrm{g} / \mathrm{ml}$ chymostatin, $5 \mu \mathrm{g} / \mathrm{ml}$ pepstatin, $50 \mathrm{mM} \mathrm{NaF}$, and $5 \mathrm{mM} \mathrm{Na} \mathrm{VO}_{4}$ at $\mathrm{pH}$ 7.4). Cdk5 was immunoprecipitated from the ventral striatal homogenates using an anti-Cdk5 antibody conjugated to agarose beads (C8; Santa Cruz Biotechnology). The immunoprecipitated samples were resuspended and incubated with $2 \mu \mathrm{g}$ of histone $\mathrm{H} 1$ (type II-S) (Sigma Co.) in $20 \mu \mathrm{l}$ of kinase assay buffer $(50 \mathrm{mM}$ HEPES, $10 \mathrm{mM} \mathrm{MgCl}_{2}, 1 \mathrm{mM}$ DTT, $50 \mu \mathrm{M}$ ATP, and $5 \mu \mathrm{Ci}$ $\left.\left[\gamma-{ }^{32} \mathrm{P}\right] \mathrm{ATP}\right)$ for $10 \mathrm{~min}$ at $30^{\circ} \mathrm{C}$. The reaction was terminated by spotting $20 \mu \mathrm{l}$ of the reaction mixture on a P81 phosphocellulose disc (Whatman P81, Upstate Biotechnology Inc.) or by adding $5 \mu \mathrm{l}$ of $5 \times$ sample buffer $(250 \mathrm{mM}$ Tris- $\mathrm{HCl}, 40 \%$ glycerol, $8 \%$ SDS, and 20\% 2-mercaptoethanol at $\mathrm{pH} 6.8$ ). The discs were washed three times ( $5 \mathrm{~min}$ each) with $0.75 \%$ phosphoric acid, followed by soaking in $95 \%$ ethanol for $5 \mathrm{~min}$ and dried in the air. The radioactivity was measured with a scintillation counter (Packard Inc.) and corrected with basal activity, measured at the corresponding time but with boiled samples. Alternatively, the samples were separated by SDS-PAGE (15\% polyacrylamide gels). After the samples were resolved, the gels were dried and radiographic bands were generated and quantified with a PhosphorImager system (Typhoon 9200; Amersham Pharmacia Biotech).

\section{Experimental Protocols}

For characterization of acute METH-induced behaviors, one set of rats was injected systemically with either $4 \mathrm{mg} / \mathrm{kg}$ METH or normal saline, while another set of rats received a bilateral intra-accumbens injection of roscovitine $(10 \mathrm{mM})$ or vehicle $4 \mathrm{~h}$ prior to systemic injection of $4 \mathrm{mg} / \mathrm{kg} \mathrm{METH}$. Immediately after the METH injection, all the rats were transferred to the behavioral monitor and their locomotor activity and stereotypy were recorded. For chronic METHinduced behavioral sensitization, rats were injected with $4 \mathrm{mg} / \mathrm{kg}$ METH i.p. once daily for 14 consecutive days. Control rats received daily normal saline injection. At withdrawal day 7 , one set of chronic METH- or salinetreated rats was challenged with $1 \mathrm{mg} / \mathrm{kg} \mathrm{METH}$, while another set of METH-treated rats received a bilateral intraaccumbens injection of roscovitine $(10 \mathrm{mM})$ (or vehicle) $4 \mathrm{~h}$ prior to challenge with $1 \mathrm{mg} / \mathrm{kg}$ METH. Rats were recorded immediately for the amount of locomotor activity and stereotypy every $5 \mathrm{~min}$ for a total session of $2 \mathrm{~h}$.

To assess the biochemical changes after acute METH administration, another set of rats was injected systemically with $4 \mathrm{mg} / \mathrm{kg}$ METH and killed by decapitation after 1, 2, 4, 8 , and $24 \mathrm{~h}$ ( $n=3-5$ for each time point). The control rats were injected with normal saline at the corresponding times. To compare the biochemical alterations between chronic METH and saline groups, both groups of rats were killed at drug withdrawal day 7 without any behavioral measurements. To validate the behavioral effect of roscovitine, animals were killed $2 \mathrm{~h}$ after $1 \mathrm{mg} / \mathrm{kg}$ METH challenge. In those animals, tissues from the ventral striatum were dissected for the designated biochemical analyses.

\section{Statistical Analysis}

All the data were analyzed with the program GraphPad Prism $^{\text {TM }}$. Results are expressed as mean values \pm SEM. Behavioral and biochemical data were analyzed either by a one- or two-way ANOVA followed by the post hoc Dunnett's multiple comparison test or a nonpaired Student's $t$-test. The level of statistical significance was set at $P<0.05$.

\section{RESULTS}

\section{Effect of Acute METH on Cdk5 Activity in the Ventral Striatum}

As shown in Figure 1, acute METH $(4 \mathrm{mg} / \mathrm{kg})$ injection in the rats significantly induced Cdk5 activity in the ventral striatum in a time-dependent manner. The activity of Cdk5 appeared to increase at $1 \mathrm{~h}$ (approximately 50\%), with maximal stimulation observed at $2 \mathrm{~h}$ after METH administration $(P<0.05)$, remained relatively elevated until $8 \mathrm{~h}$, and returned to its basal level after $24 \mathrm{~h}$. To confirm the Cdk5 activation by acute METH treatment, $\left[\gamma-{ }^{32} \mathrm{P}\right]$ histone $\mathrm{H} 1$ samples were resolved in SDS-PAGE. The results displayed a 


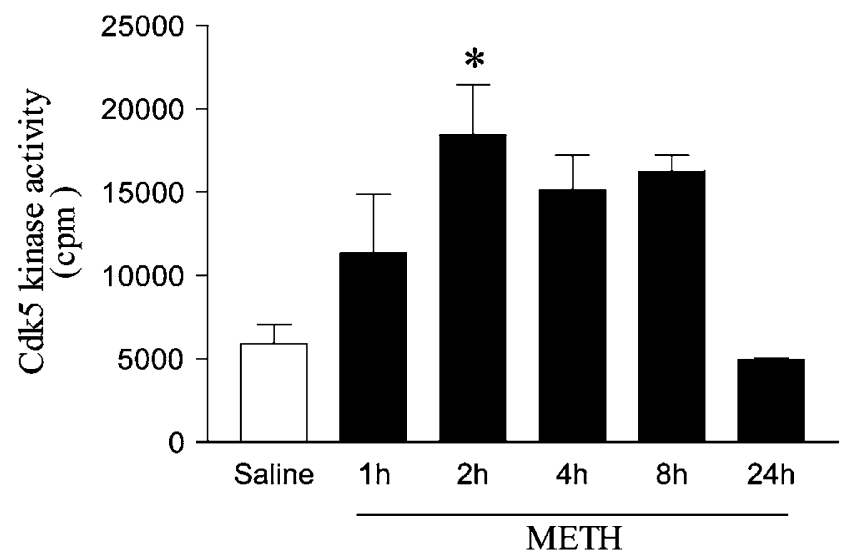

Figure I Time course study of acute METH-induced in vitro Cdk5 activity in the ventral striatum. The numbers indicate hours after saline or METH $(4 \mathrm{mg} / \mathrm{kg})$ injection. The samples from saline-treated rats at different time periods displayed no difference and were pooled. Cdk5 activity was expressed as specific counts (cpm). Each bar represents six samples per group. ${ }^{*} P<0.05$ compared to the saline-treated group.

similar pattern of Cdk5 activation as revealed by radioactivity counts, with maximal $\left[\gamma-{ }^{32} \mathrm{P}\right]$ incorporation at $2 \mathrm{~h}$ post-METH administration and progressively decreased until $24 \mathrm{~h}$ (data not shown). However, acute METH-induced Cdk5 activation was unrelated to its protein expression since the total amount of Cdk5 in the ventral striatum remained constant until $24 \mathrm{~h}$ after injection of $4 \mathrm{mg} / \mathrm{kg}$ METH (Figure 2a).

\section{Effect of Acute METH on Cdk5 Activator, p35 Protein in} the Ventral Striatum

Since most brain Cdk5 activation requires the counterpart p35 and/or p39 protein translocation (Dhavan and Tsai, 2001 ), we analyzed the amount of p35 protein in both the membrane and cytosolic fractions from the ventral striatum of control and METH-treated rats. Acute METH treatment did not affect the total amount of p35 for up to $8 \mathrm{~h}$ (Figure 2a). Nevertheless, we observed a significant increase in the membrane form of p35 after METH injection, with maximal elevation occurring at $2 \mathrm{~h}$ post-METH $(P<0.01)$ and returning to the basal level after $8 \mathrm{~h}$ (Figure $2 \mathrm{~b}$ ). The relatively unchanged level of p35 in the cytosolic fraction (Figure 2b) suggests that a minor, but significant, portion of p35 translocates to the membrane fraction and might be responsible for Cdk5 activation.

\section{Effect of Acute METH on DARPP-32 Phosphorylation in the Ventral Striatum}

To investigate the effect of acute treatment with METH on downstream Cdk5 signaling, we examine the phosphorylation of DARPP-32 in the ventral striatum after a single $4 \mathrm{mg} /$ $\mathrm{kg}$ METH injection. Since METH-evoked dopamine would act on dopamine $D_{1}$ receptors and trigger DARPP-32 phosphorylation at Thr34, while Cdk5 activation could phosphorylate DARPP-32 at Thr75, we, therefore, examined the amount of DARPP-32 phosphorylation at either Thr34 or Thr75 residues. The results indicate that acute METH differentially regulated DARPP-32 phosphorylation at both Thr34 and Thr75 positions, without changes in the total amount of DARPP-32 in the ventral striatum (Figure 2c). The phosphorylation of DARPP-32/Thr34 significantly enhanced at approximately $1-2 \mathrm{~h}$ post-METH $(P<0.001)$ indicates a fast signaling possibly through $\mathrm{D}_{1}$-PKA pathway. On the other hand, the phosphorylation of DARPP-32/ Thr75, although statistically insignificant, had a tendency to increase at $1 \mathrm{~h}$ after METH injection and sustained for up to $8 \mathrm{~h}$ reflects somewhat the result of Cdk5 activation. Overall, the results implicate that both $\mathrm{D}_{1}$-PKA and intracellular Cdk5 signaling were regulated by the acute METH treatment.

\section{Effect of Cdk5 Inhibitor, Roscovitine on Acute METH-Evoked Behavioral Activation}

Since we had determined that METH could activate Cdk5 activity and p35 translocation, we intend to test if Cdk5 activation was associated with METH-evoked behavioral effects. As shown in Figure 3, $4 \mathrm{mg} / \mathrm{kg}$ METH increased both locomotor activity and stereotypy compared to the saline control $(\mathrm{F}(2,522)=290.1, P<0.001$ and $\mathrm{F}(2,522)=145.7$, $P<0.001$, respectively), and both cumulative counts reached a significant level during the first hour $(P<0.001$ and 0.01 , respectively; Figure 3, inset). Intra-accumbens injection of the Cdk5 inhibitor roscovitine $(10 \mathrm{mM} /$ each side) significantly inhibited the locomotor activity $(P<0.01)$, but mildly suppressed the stereotypy (50-100 min post-METH) in the METH-treated groups, indicating that Cdk5 signaling participates in the acute METH-induced behavioral activation. The administration of roscovitine alone in the nucleus accumbens did not evoke any behavioral responses in naive animals (data not shown). The histological sections with crystal violet staining confirmed the location of the cannula tip in the nucleus accumbens-shell region (Figure 9b).

\section{Cdk5 Activity and p35 Translocation in the Ventral Striatum of Control and METH-Sensitized Rats}

The finding that acute METH activates Cdk5 signaling prompted us to investigate if chronic METH treatment, to the point that the animal develops behavioral sensitization, would affect Cdk5 activity and its downstream regulator(s). Animals treated with $4 \mathrm{mg} / \mathrm{kg} \mathrm{METH}$ for 14 consecutive days developed characteristic stereotypy sensitization as the behavioral profile of the same subjects at day 1 was compared to day $14(P<0.05$; Figure $4 \mathrm{a})$. At this relative high dosage of METH, daily locomotor activity did not increase progressively (data not shown). Further, when chronic METH- and saline-injected animals were challenged with a low-dose of METH $(1 \mathrm{mg} / \mathrm{kg})$, both horizontal locomotor activity and stereotypy were significantly higher in chronic METH-treated rats than chronic saline-treated controls $(P<0.05$; Figure $4 \mathrm{~b})$, indicating the development of behavioral sensitization. A notable biochemical change along with behavioral sensitization was the upregulation of $\mathrm{TH}$ in the ventral striatum (Figure 6a; animals killed $2 \mathrm{~h}$ after METH challenge). As compared with the chronic saline-treated controls, the basal activity of Cdk5 (both ingel and radioactivity counts of $\left[\gamma_{-}{ }^{32} \mathrm{P}\right]$ histone $\left.\mathrm{H} 1\right)$ that monitored at withdrawal day 7 in METH-sensitized rats was 
a

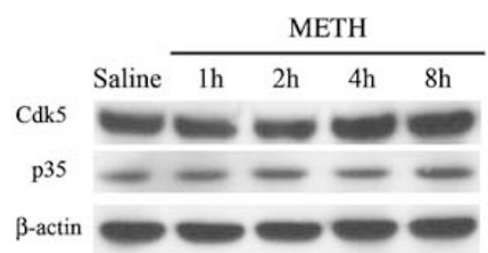

b
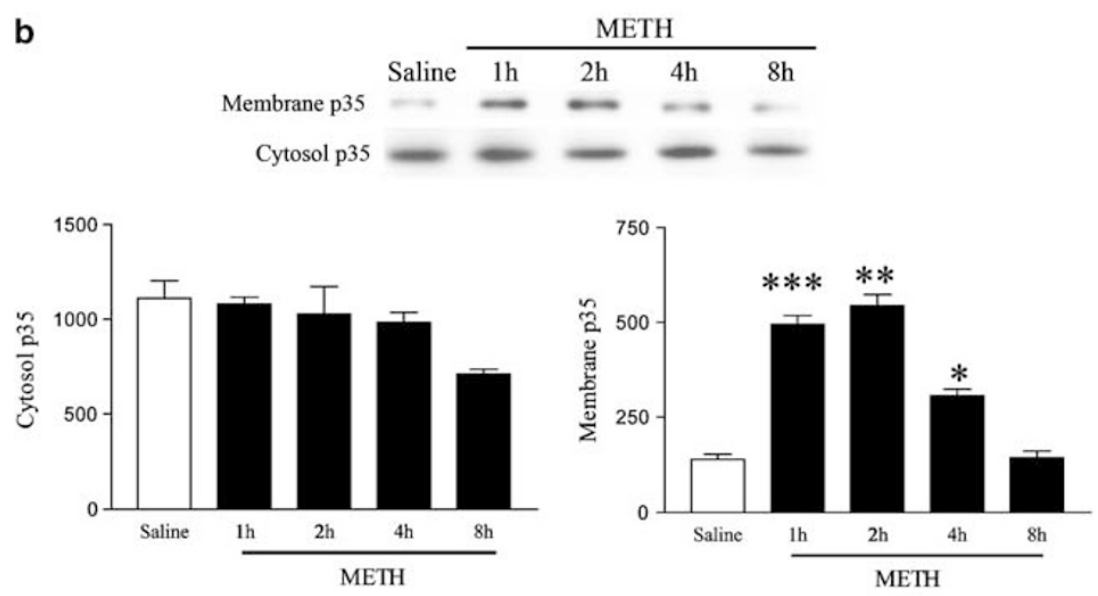

C
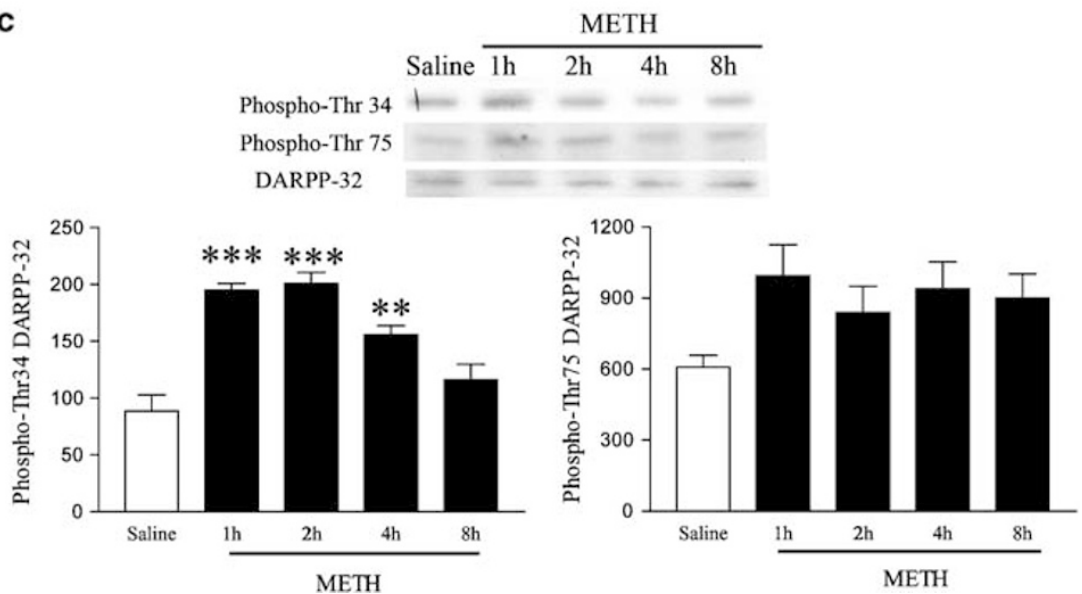

Figure 2 Representative Western immunoblots of (a) total Cdk5 and p35 protein, (b) membrane and cytosolic fractions of p35 protein and (c) phosphoDARPP-32 at Thr34 and Thr75 in the ventral striatum of acute saline- or METH-treated groups. The numbers indicate hours after saline or METH injection. The samples from saline-treated rats at different time periods displayed no difference and were pooled. Each lane represents pooled ventral striata $(n=2-3)$ and the experiment was repeated at least three times. Bar graphs illustrate quantitative measurement of membrane or cytosol fraction of p35 and phosphoDARPP-32 /Thr34 or Thr75, normalized with $\beta$-actin (b; data not shown) or DARPP-32 (c) signals for variations in protein loading ( $n=3-5$ ). $* P<0.05$; ** $P<0.0$ I; *** $P<0.00$ I compared to the corresponding saline-treated group.

significantly increased $(P<0.001$; Figure 5$)$. Similar to the acute METH treatment, chronic METH administration did not alter the total amount of Cdk5 in the ventral striatum (Figure 6a). The Cdk5 activator p35 protein displayed an apparent translocation, that is, an increase in the membrane fraction of p35 $(P<0.01)$ and a decrease in the cytosolic fraction of p35 $(P<0.05)$ during METH withdrawal in rats (Figure 6b). On the other hand, the total amount of p35 protein in the ventral striatum was similar between the chronic saline- and METH-treated groups (Figure 6a). The results on p35 protein are consistent with the Cdk5 upregulation observed in the ventral striatum of behavioral sensitized rats, indicating a persistent activation of Cdk5 signaling due to chronic METH treatment.
DARPP-32 Phosphorylation and PP-2A Expression in the Ventral Striatum of Control and METH-Sensitized Rats

Consistent with Cdk5/p35 activation, the basal DARPP-32/ Thr75 phosphorylation was higher in METH-sensitized rats than the saline-treated controls $(P<0.01$; Figure $6 \mathrm{c})$. On the other hand, the basal phosphorylation of DARPP-32/Thr34 was decreased in METH-sensitized rats compared to the saline-treated controls $(P<0.001$; Figure $6 \mathrm{c})$. Furthermore, the amount of PP-2A (catalytic subunit), a phosphatase that dephosphorylates the DARPP-32 at the Thr75 residue, decreased in the ventral striatum of METH-sensitized rats $(P<0.01)$, suggesting that a long-lasting change in DARPP- 


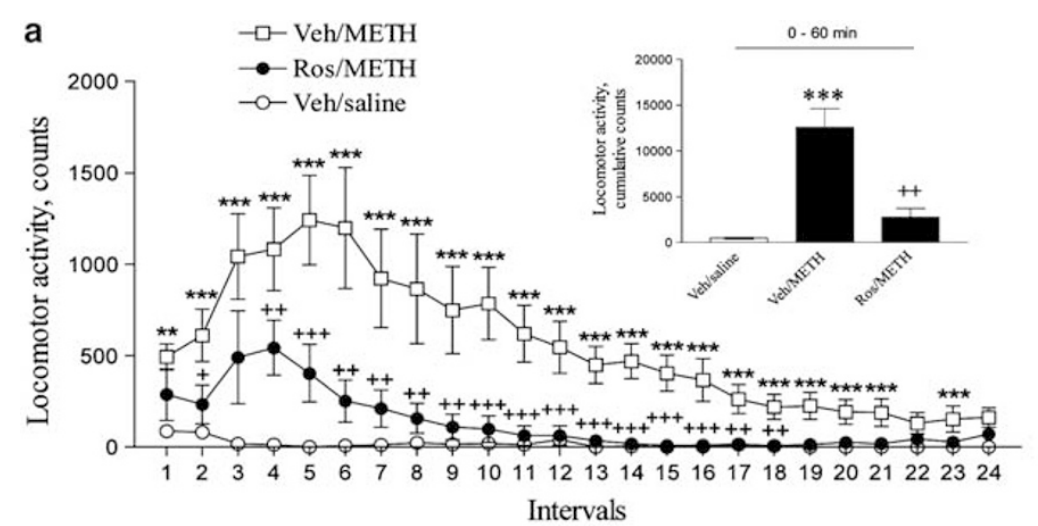

b

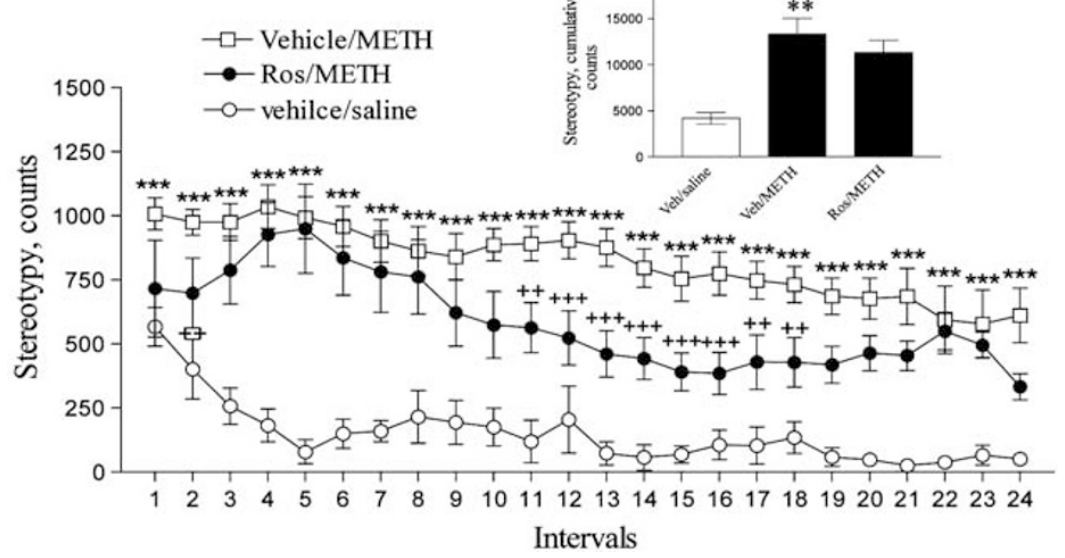

Figure 3 (a) Locomotor activity and (b) stereotypy of vehicle- or roscovitine-pretreated rats induced by acute treatment with saline or METH (4 mg/kg). Roscovitine $(10 \mu \mathrm{M})$ was injected bilaterally into the nucleus accumbens $4 \mathrm{~h}$ prior to systemic METH injection. After METH injection, counts of every 5 min interval of both behavioral parameters were recorded for a total session of $2 \mathrm{~h}$ ( $n=5-7 /$ group). The bar graphs indicate the cumulative counts of both behaviors at the first hour after METH treatment. Two-way ANOVA (treatment $\times$ time, with repeated measures over time) indicated there were significant treatment $\times$ time interaction for locomotor activity and stereotypy $(F(46,522)=10.10$ and 6.28 , respectively; $P<0.00 I)$. Veh, vehicle; Ros, roscovitine. **P $<0.01 ; * * * P<0.001$, the Veh/METH group compared to the Veh/Saline group at corresponding time intervals or cumulative counts. ${ }^{+} P<0.05$; ${ }^{++}$ $P<0.01 ;{ }^{+}+{ }^{+} P<0.001$, the Ros/METH group compared to the Veh/METH group at corresponding time intervals or cumulative counts (Dunnett's comparison test).

32/Thr75 signaling resulted from chronic METH treatment (Figure 6a).

\section{Effects of Intra-Accumbal Roscovitine on Behavioral Sensitization to METH}

The finding of enhanced Cdk5 activity in METH-sensitized rats prompted us to test if $\mathrm{Cdk} 5$ participates in the development of behavioral sensitization. When chronic METH-treated rats received a bilateral injection of $10 \mathrm{mM}$ roscovitine in the nucleus accumbens at drug withdrawal day 7, the locomotor sensitization induced by $1 \mathrm{mg} / \mathrm{kg}$ METH was significantly reduced $(P<0.01$; Figure 7$)$. On the other hand, the treatment with roscovitine in the nucleus accumbens did not affect the sensitized stereotypy induced by METH. The histological verification indicated that the cannula tip was located in the nucleus accumbens-shell region (Figure 9b). In general, we noticed an overall reduction in total locomotor activity $(-45 \%)$ and stereotypy $(-35 \%)$ counts in these animals as compared to previous groups (cumulative counts in Figure 4 vs Figure 7), possibly due to the acute surgical procedure or response from different sets of animals. To confirm the effects of roscovitine, we measured the Cdk5 activity in drug-treated control and METH groups after the behavioral test. The treatment with roscovitine significantly inhibited the Cdk5 activity in behavior-sensitized rats $(P<0.001$; Figure $8 \mathrm{a})$, which validates the behavioral effects of roscovitine. In the same preparation, roscovitine effectively suppressed the amount of DARPP-32/Thr75 phosphorylation $(P<0.001$; and, in contrast, increased the amount of the DARPP-32/ Thr34 signal in the ventral striatum $(P<0.001$; Figure $8 \mathrm{~b})$. The total amount of $\beta$-actin in the ventral striatum was not significantly different between the two groups of animals (Figure 8b).

\section{DISCUSSION}

We have demonstrated that acute and chronic METH treatment can induce Cdk5 activity as well as p35 translocation in the ventral striatum. In addition, Cdk5 and DARPP-32 signaling in the ventral striatum could participate in the development of METH sensitization, since 
$\mathbf{a}$

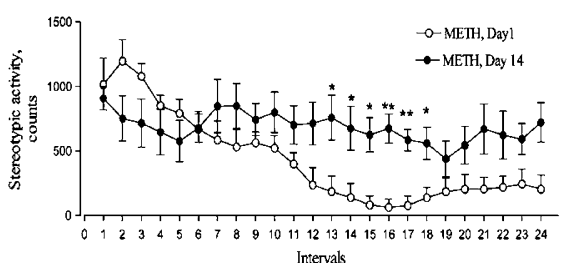

Irtervals

b

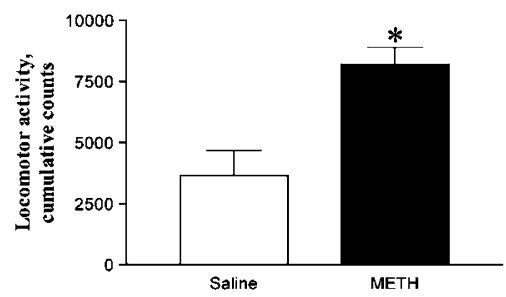

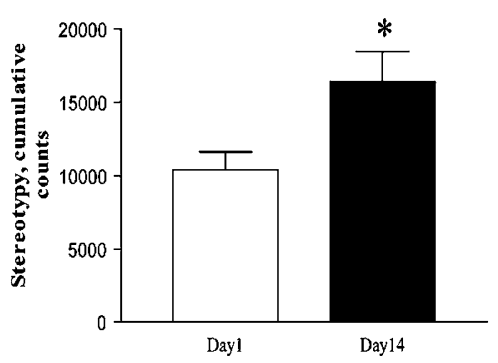

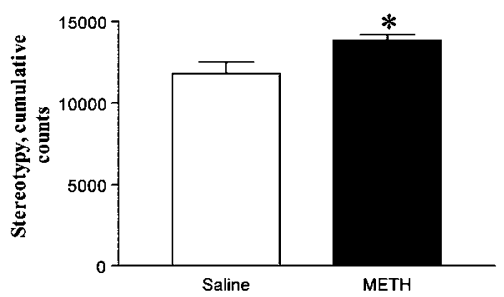

Figure 4 (a) METH (4 mg/kg) induced stereotypy at day I and day 14. Counts of every $5 \mathrm{~min}$ interval were recorded for a total session of $2 \mathrm{~h}$ ( $\mathrm{n}=6 /$ group). The bar graphs indicate the cumulative counts of $2 \mathrm{~h}$. Two-way ANOVA (treatment $\times$ time, with repeated measures over time) indicated there were significant treatment $\times$ time interaction $(F(24,120)=5.33$, respectively; $P<0.00 I)$. (b) Counts of locomotor activity and stereotypy of the chronic saline- or METH-treated rats induced by I mg/kg METH at withdrawal day 7. Bar graphs indicate the cumulative counts of the corresponding behaviors recorded for $2 \mathrm{~h}$ after a challenge with $\mathrm{METH}(n=8,8)$. $* P<0.05$; $* * P<0.0 \mathrm{I}$, compared to METH-treated animals of day I at corresponding time intervals or cumulative counts of day I or saline control (Dunnett's comparison test).

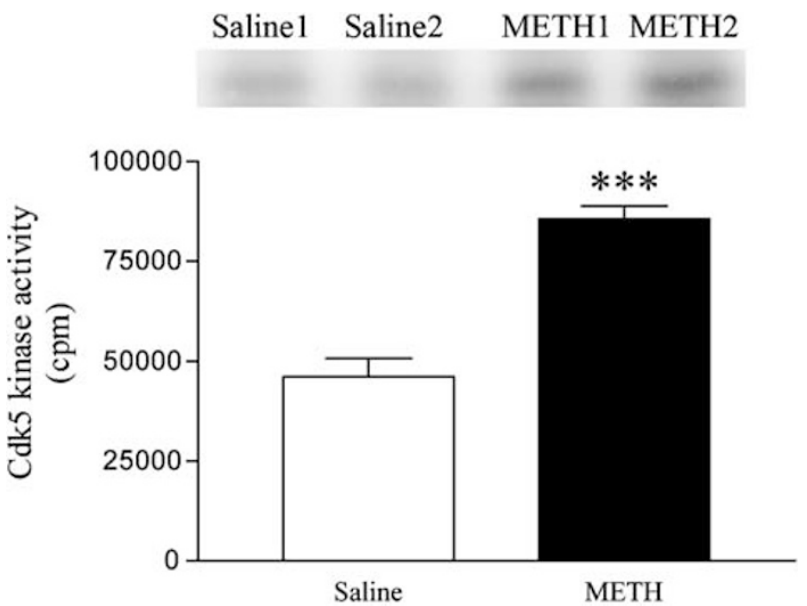

Figure 5 In vitro Cdk5 activity of the chronic saline- and METH-treated groups measured at drug withdrawal day 7 without a challenge with METH. Duplicate $\left[\gamma^{32} \mathrm{P}\right]$ histone samples resolved by SDS-PAGE, and radioactivity counts generated from the disc. Each lane represents pooled ventral striata $(n=2-3)$ and the experiment was repeated at least three times. Each bar represents six samples per group. $* * * P<0.001$ compared to the salinetreated group.

an intra-accumbens injection of roscovitine, inhibited Cdk5 activity and DARPP-32/Thr75 phosphorylation and suppressed the behavioral upregulation in METH-sensitized rats. Consistent with these observations, we noted a concomitant change in the DARPP-32/Thr34 signal in both the acute and chronic preparations, and a decrement of PP$2 \mathrm{~A}$ levels in the ventral striatum of behavior-sensitized rats. The results clearly indicate that Cdk5/p35 and DARPP-32 signal in the ventral striatum plays a significant role in the acute and chronic effects of METH.

We have found that acute METH treatment transiently increases the Cdk5 activity in the ventral striatum, with a minor increase at $1 \mathrm{~h}$ and peak activation occurring approximately at $2 \mathrm{~h}$ after drug administration and returning to the basal level after $24 \mathrm{~h}$. The Cdk5 upregulation appears to parallel the translocation of its activator, p35 protein, from the cytosolic fraction to the membrane, since maximal levels of p35 in the membrane fraction occurred at $2 \mathrm{~h}$ after acute METH injection. These findings are consistent with the previous view that brain Cdk5 usually associated with its regulatory partner, $\mathrm{p} 35$ protein, via Nterminal myristoylation anchors on the cell membrane, to exert its kinase activity (Lew, 1994; Amitabh and Tsai, 2001). However, we notice membrane form of p35 returned to basal level at $8 \mathrm{~h}$ after METH, while at this time, the Cdk5 activity remained elevated. Although speculative, it is possible that another cellular mediator(s) could be involved in the sustained activation of Cdk5. It was reported that casein kinase I could phosphorylate Cdk5 at Ser159 residue, leading to Cdk5 activation (Grant et al, 2001; Sharma et al, 1999); whether casein kinase I also plays a role in METHinduced Cdk5 activation requires further investigation. In this study, acute METH treatment did not affect the total amount of Cdk5 and p35 proteins; besides there was no concomitant decrease in cytosolic p35 protein after acute METH injection, possibly due to the small, but significant, fraction of p35 translocated to the cell membrane during acute drug treatment. A similar pattern of Cdk5 activation but more pronounced p 35 translocation was also observed in the ventral striatum of chronic METH-treated rats, while the total amount of Cdk5 and p35 remained the same. Since considerable Cdk5 activation and p35 translocation were recorded at withdrawal day 7 without drug challenge, the results suggest that the robust $\mathrm{Cdk} 5 / \mathrm{p} 35$ alteration was affected by the repetitive METH treatment.

It is well established that Cdk5 is involved in both neurodevelopment and neurodegeneration and is known to regulate the cytoskeleton, axonal guidance, membrane 
a
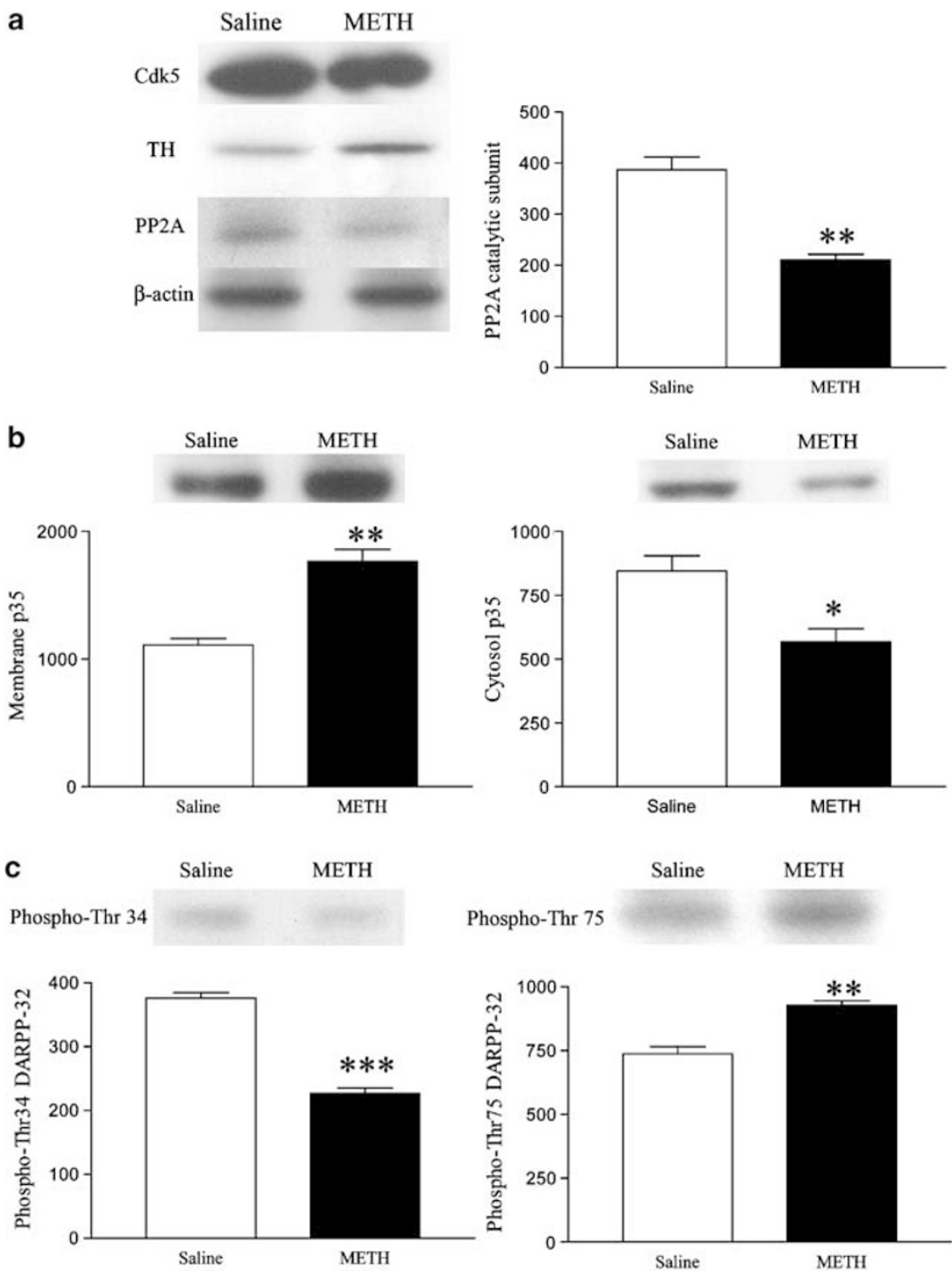

Figure 6 Representative Western immunoblots of (a) total Cdk5, TH, and protein phosphatase PP-2A, (b) membrane and cytosolic fractions of p35 protein, and (c) phospho-DARPP-32 at Thr34 and Thr75 in the ventral striatum of the chronic saline- or METH-treated groups without a challenge with METH. The experiment was repeated at least three times. The bar graphs indicate quantitative counts of PP2A, membrane, or cytosol fraction of p35 and phospho-DARPP-32 /Thr34 or Thr75, normalized with $\beta$-actin ( $a$ and b) or DARPP-32 (c) signals for variations in protein loading ( $n=3-5$ ).

transport, synaptic function, dopamine signaling, and drug addiction (Dhavan and Tsai, 2001). A number of proteins with diverse functions have been identified as substrates for Cdk5, including $\mathrm{p} 35 / \mathrm{p} 39$, Tau protein, DARPP-32, PP-1 inhibitor, and synapsin 1 (Smith et al, 2001). Activation of Cdk5 would phosphorylate DARPP-32 at Thr75 and then suppress the PKA activity on DARPP-32 (phosphorylate Thr34) (Greengard, 2001). Therefore, Cdk5 and PKA seem to exert opposing effects on dopaminergic signaling through phosphorylation of DARPP-32 and possibly other proteins in the pathway (Nishi et al, 2000). In both acute and chronic METH preparations, we also observed changes in DARPP-32 phosphorylation at both Thr34 and Thr75 in the ventral striatum. The maximal phosphorylation of DARPP-32 at Thr34 occurred at $1 \mathrm{~h}$ after acute METH treatment; however, for DARPP-32/Thr75, a plausible increase occurred between $1-2 \mathrm{~h}$ and lasted until $8 \mathrm{~h}$. We speculate that in the acute METH treatment, the fast
DARPP-32/Thr34 phosphorylation in the ventral striatum could result from $D_{1}$ dopamine receptor activation, but the signal is diminished during the later hours possibly by the subsequent Cdk5 activation and DARPP-32/Thr75 phosphorylation. On the other hand, the conceivable increase in DARPP-32/Thr75 phosphorylation relatively matches with the Cdk5 activation, further confirming Cdk5/DARPP-32/ Thr75 signaling induced by acute METH treatment. This signal cross-talk at the DARPP-32 level clearly encodes in the chronic METH preparation, since we noticed an increased basal level of DARPP-32/Thr75 phosphorylation and a decreased DARPP-32/Thr34 phosphorylation in the ventral striatum of the chronic METH-treated group. The results agree with the conclusion drawn by Greengard (Greengard, 2001) that chronic cocaine-induced Cdk5 and DARPP-32/Thr75 upregulation could be viewed as a negative feedback mechanism to suppress the dopamine $\mathrm{D}_{1}$-PKA-DARPP-32/Thr34 signaling. The data, however, 
a

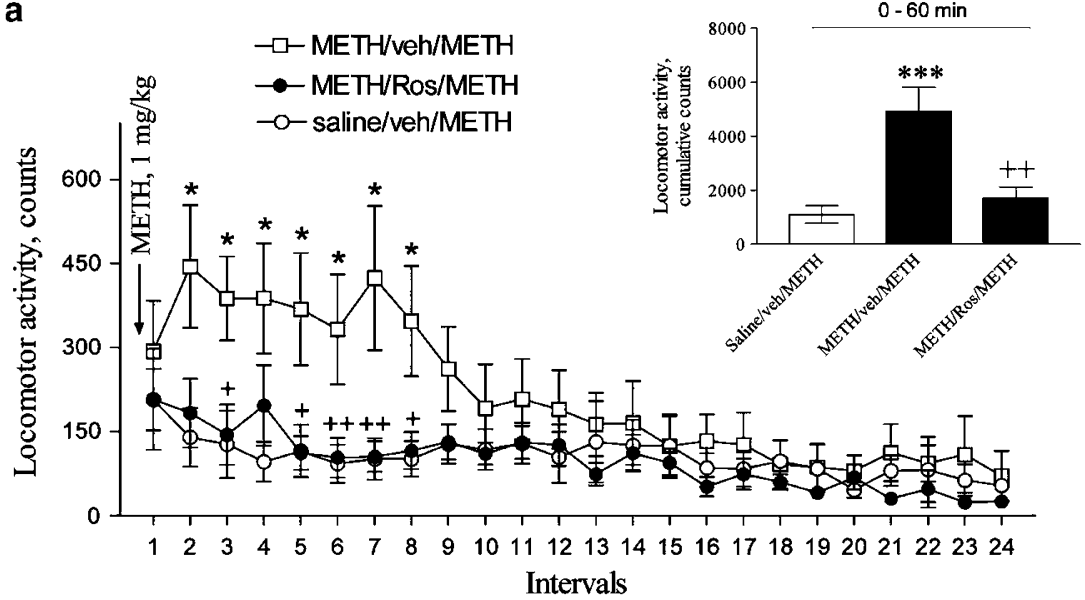

b

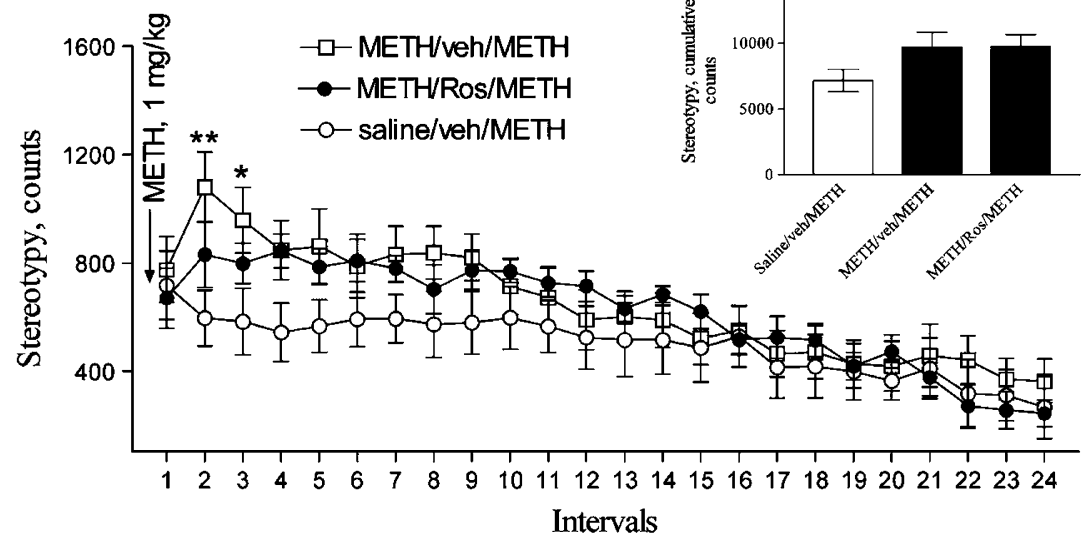

Figure 7 (a) Locomotor activity and (b) stereotypy of vehicle- or roscovitine-pretreated rats induced by acute METH (I mg/kg) challenge. Animals were chronically treated with either $4 \mathrm{mg} / \mathrm{kg} \mathrm{METH}$ or saline for 14 consecutive days. Roscovitine $(10 \mu \mathrm{M})$ was injected bilaterally into the nucleus accumbens $4 \mathrm{~h}$ prior to systemic METH injection. Every 5 min interval after METH injection, counts of both behavioral parameters were recorded for a total session of $2 \mathrm{~h}$ ( $n=8$-10/group). The bar graphs indicate the cumulative counts of both behaviors at the first hour after METH treatment. Two-way ANOVA (treatment $\times$ time, with repeated measures over time) indicated that there was significant treatment $\times$ time interaction for locomotor activity and stereotypy $(F(46,336)=13.16$ and 5.90, respectively; $P<0.00 \mathrm{I})$. Veh, vehicle; Ros, roscovitine. $* P<0.05 ; * * P<0.0 \mathrm{I} ; * * * *<<0.00 \mathrm{I}$, the METH/veh/METH group compared to the Saline/veh/METH group at corresponding time intervals or cumulative counts. ${ }^{+} P<0.05 ;{ }^{+}+P<0.01$, the METH/Ros/METH group compared to the METH/veh/METH group at corresponding time intervals or cumulative counts (Dunnett's multiple comparison test).

disagree with the report by Bibb et al (2001) in that they observed an increase in the amount of Cdk5 after repeated cocaine administration. Although the impact on DARPP-32 signaling is similar, the discrepancy between cocaine and METH treatments could possibly result from a different mode of action on targeted dopamine transporter or different source of tissues (caudate/putamen in Bibb's study $v s$ nucleus accumbens in this experiment). On the other hand, the difference could also be due to the route of administration since Cdk5 levels remained the same following repeated cocaine self-administration ( $\mathrm{Lu}$ et al, 2003). Interestingly, we observed a decrease in the amount of PP-2A catalytic subunit in the ventral striatum of chronic METH-treated rats. Activation of PP-2A, possibly via the dopamine $\mathrm{D}_{1}$-PKA pathway, was reported to dephosphorylate DARPP-32 at Thr75, and, thus, reduce the Cdk5/ DARPP-32/Thr75 signaling (Bibb et al, 1999; Greengard, 2001). Alternatively, PP-2A can be directly stimulated by the action of NMDA/AMPA receptors (Nishi et al, 2002), an event that could possibly occur in METH-sensitized rats.
Our observation, hence, provides evidence that increased Cdk5 activity and decreased PP-2A after chronic METH treatment would synchronize the dopaminergic and glutamatergic transmission in the ventral striatum, in addition dually potentiating the DARPP-32/Thr75 signaling.

Consistent with our biochemical observations that acute METH evoked Cdk5 activity and p35 translocation, the Cdk5 inhibitor roscovitine, given acutely into the nucleus accumbens, significantly suppressed METH-enhanced locomotor activity. The profound roscovitine effect on locomotion, but less on stereotypy, indicates that the intracranial drug injection was confined to the nucleus accumbens, a region responsible for the locomotor activation (Kuczenski and Segal, 1999). Both biochemical and behavioral results lead us to speculate that, other than the dopamine $\mathrm{D}_{1}$ receptor-PKA pathway, Cdk5/p35 and the downstream signaling (the DARPP-32/Thr75 and/or other substrate(s)) in the ventral striatum participate in the acute METH-evoked behavioral excitation. To our knowledge, this is the first report that associates the acute 


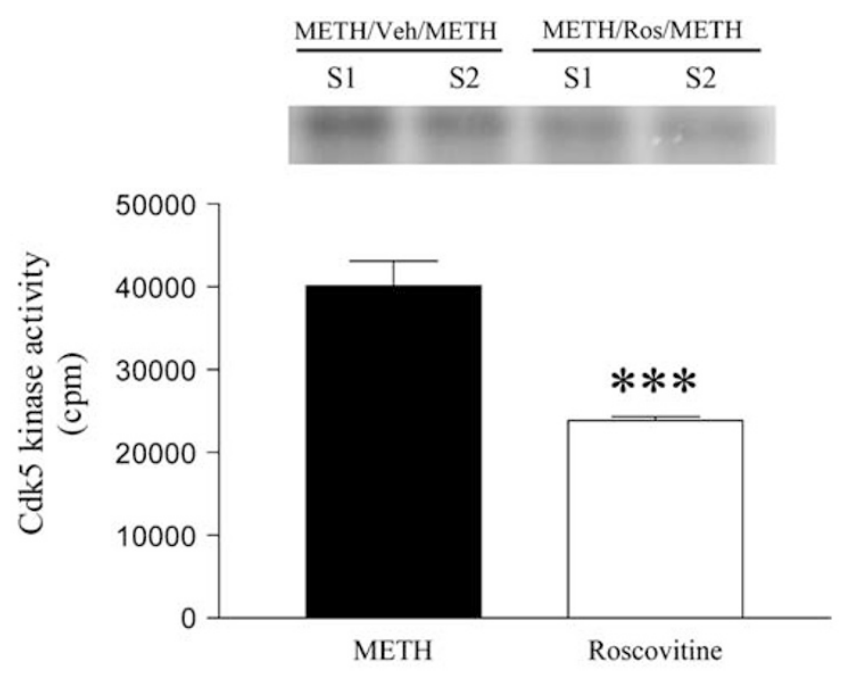

b

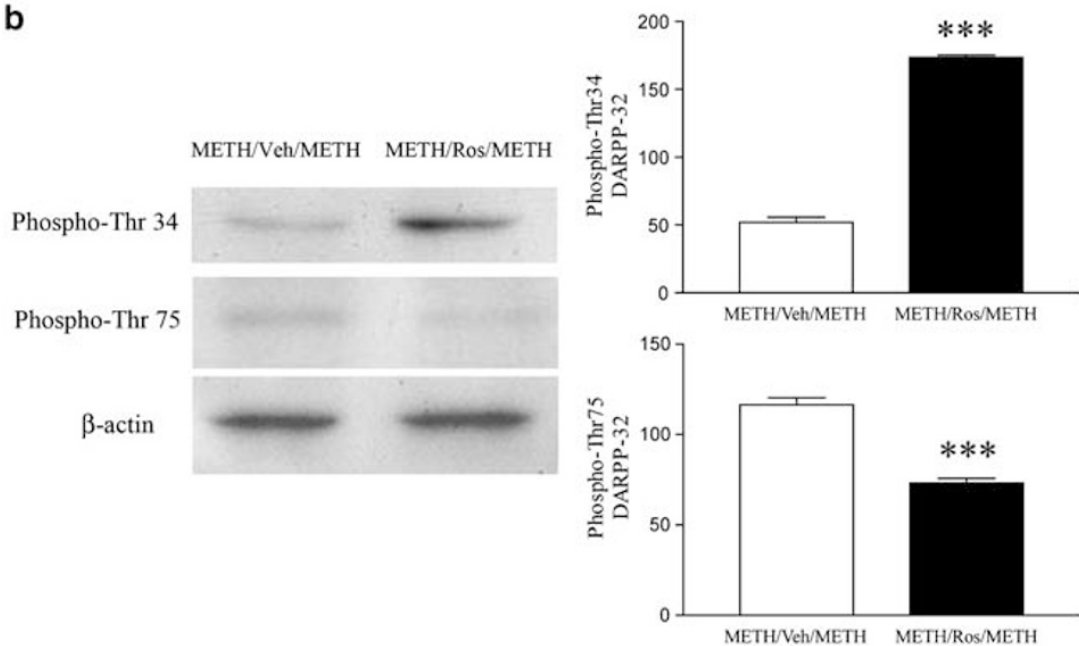

Figure 8 (a) In vitro Cdk5 activity (duplicate $\left[\gamma^{32} \mathrm{P}\right]$ histone samples resolved by SDS-PAGE, and radioactivity counts generated from the disc) and (b) Western immunoblots of phospho-DARPP-32 at Thr34 and Thr75 in the ventral striatum of the chronic METH-injected rats with (METH/Ros) or without roscovitine (METH/Neh) treatment and challenged with METH at withdrawal day 7 . The animals were chronically treated with $4 \mathrm{mg} / \mathrm{kg} \mathrm{METH}$ for 14 consecutive days. Roscovitine $(10 \mu \mathrm{M})$ was injected bilaterally into the nucleus accumbens $4 \mathrm{~h}$ prior to systemic challenge with I mg/kg METH. Each lane represents pooled ventral striata $(n=2-3)$ and the experiment was repeated at least three times. The bar represents six samples per group. In (b), the $\beta$ actin signals were normalized for variations in protein loading. $* * * P<0.00$ I compared to the METH/Veh/METH group.

behavioral and biochemical effects of psychostimulant with Cdk5 activity.

Other than acute behavioral excitation, repetitive METH administration would lead to behavioral sensitization. In behavior-sensitized animals, a cascade of neuroadaptations, including altered levels of G-proteins, cAMP, TH, and neurofilaments (Nestler and Aghajanian, 1997), were identified primarily in the nucleus accumbens (Cador et al, 1999; Vanderschuren and Kalivas, 2000). In our model system, we did observe a characteristic behavioral supersensitivity as well as TH upregulation in the ventral striatum (similar to the report of Todtenkopf and Stellar, 2000), indicating the establishment of behavioral sensitization to METH. When METH-sensitized rats were exposed to a Cdk5 inhibitor, roscovitine, in the nucleus accumbens, the behavioral supersensitivity, induced by a low dose of METH, was significantly suppressed (to an extent of acute METH-induced locomotor response). The result could generally reflect an acute inhibitory effect of roscovitine on METH-induced behavioral activation, and alternatively the data might suggest that Cdk5/p35 signaling in the ventral striatum could participate in the behavioral expression during chronic METH treatment. Previously, Bibb et al (2001) reported an escalated locomotor behavioral response after roscovitine treatment in chronic cocaine-treated rats. It is possible that the apparent behavioral discrepancy between the roscovitine treatments is due to the differential role of Cdk5 during the initiation ('inhibitory'; Bibb et al) vs expression ('stimulatory'; our observation) stage of the development of psychostimulant addiction.

Repetitive cocaine or amphetamine exposure could increase the number of dendritic spines of medium spiny neurons in the nucleus accumbens as well as of the pyramidal neurons in the medial prefrontal cortex (Robinson and Kolb, 1997, 1999). Interestingly, cocaine-induced proliferation of dendritic spines in the nucleus accumbens depends on the activity of Cdk5 (Norrholm et al, 2003). Cdk5 is also known to interact, directly or indirectly, with a 

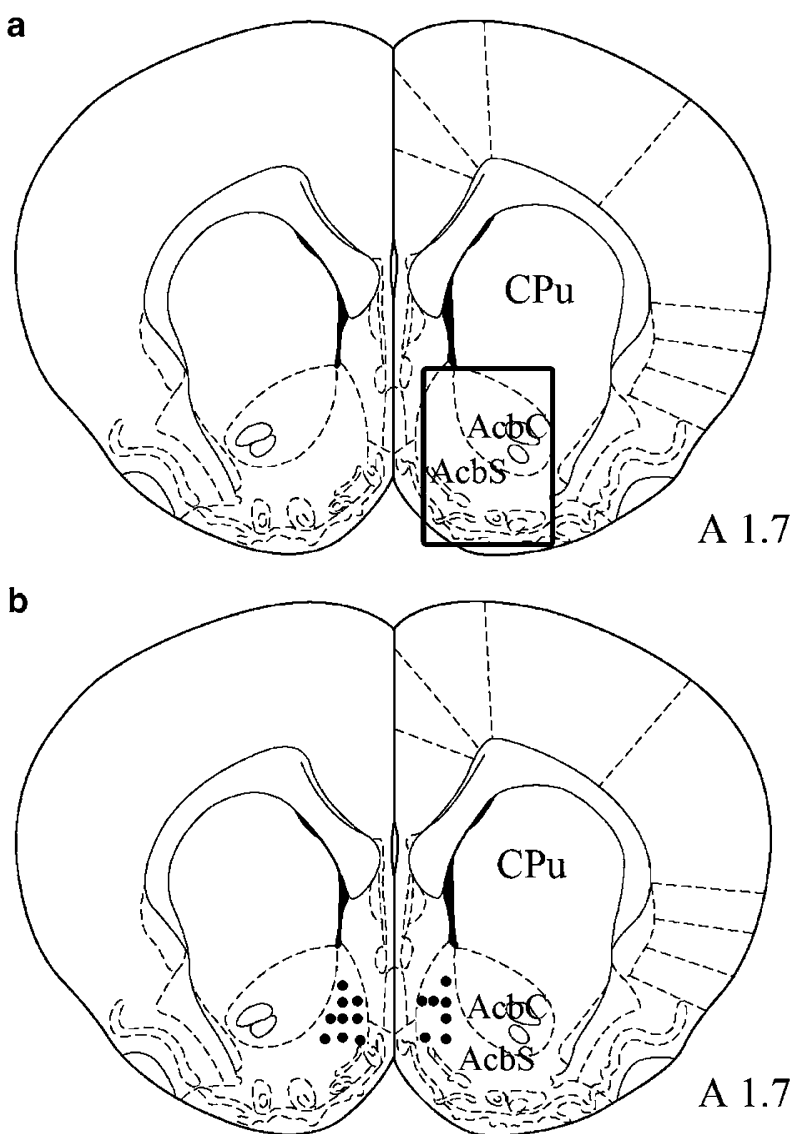

Figure 9 The schematic diagram illustrates (a) the dissection of ventral striatum (nucleus accumbens shell and core, $A c b C / A c b S$ ). A block of approximately $2 \mathrm{~mm}^{3}$ (L0.5-L2.5, A0.7-AI.7 with $1.0 \mathrm{~mm}$ height) was taken out as the ventral striatum. (b) Cannula tip locations in the nucleus accumbens-shell of acute (right side) and chronic (left side) roscovitinetreated animals. The diagram shows the representative coronal section in the microinjection experiment. The number in (a) indicates millimeters rostral to bregma, according to the atlas of Paxinos and Watson (1996). $\mathrm{CPu}$, nucleus caudate/putamen.

variety of actin- and microtubule-associated proteins, proteins that are involved in endocytosis and exocytosis and proteins that participate in adhesion pathways (Smith and Tsai, 2002). We speculate that chronic METH-induced Cdk5 activity would act, directly or indirectly, on those structural proteins, causing altered neurotransmission and neuroplasticity in the ventral striatum and/or prefrontal cortex. These neurochemical and anatomical changes might play an important role in the development of behavioral sensitization to METH. Overall, the present results indicate that Cdk5/p35 and DARPP-32 signaling participate in the acute and chronic METH-induced behavioral and cellular events. Nevertheless, questions such as what are the upstream regulators of Cdk5 and, in particular, changes of PP-2A in the ventral striatum after chronic treatment with METH remains to be clarified.

\section{ACKNOWLEDGEMENTS}

We thank Dr Jerome L Maderdrut of Tulane University for his assistance in editing this manuscript, and Drs Angus C Nairn and Paul Greengard of Rockefeller University for generously providing the anti-DARPP-32/Thr34 and antiDARPP-32/Thr75 antibodies. The study was supported by Grants NSC90-2314-B-182A-139 from the National Science Council and CMRP1375 from Chang-Gung Memorial Hospital, Taiwan, ROC.

\section{REFERENCES}

Amitabh G, Tsai LH (2001). A kinase to dampen the effects of cocaine? Science 292: 236-239.

Bibb JA, Chen JS, Taylor JR, Svenningsson P, Nishi A, Snyder GL et al (2001). Effects of chronic exposure to cocaine are regulated by the neuronal protein Cdk5. Nature 410: 376-380.

Bibb JA, Synder GL, Nishi A, Yan Z, Meijer L, Fienberg AA et al (1999). Phosphorylation of DARPP-32 by cdk5 modulates dopamine signaling in neurons. Nature 402: 669-671.

Cador M, Bjijou Y, Stinus L (1995). Evidence of a complete independence of the neurobiological substrates for the induction and expression of behavioral sensitization to amphetamine. Neuroscience 65: 385-389.

Cador M, Bjijou Y, Stinus L (1999). d-Amphetamine-induced behavioral sensitization: implication of a glutamatergic medial prefrontal cortex-ventral tegmental area innervation. Neuroscience 94: 705-711.

Chiang YC, Chen PC, Chen JC (2003). Dopamine $\mathrm{D}_{3}$ receptor down-regulation and functional desensitization in the olfactory tubercle of amphetamine-sensitized rats. Brain Res 972: 159-167.

Dhavan R, Tsai LH (2001). A decade of CDK5. Nat Rev 2: 749-759.

Ferrer-Alcon M, Harpe RL, Guimon J, Garcia-Sevilla JA (2003). Downregulation of neuroal $\mathrm{cdk} 5 / \mathrm{p} 35$ in opioid addicts and opiate-treated rats: relation to neurofilament phosphorylation. Neuropsychopharmacology 28: 947-955.

Grant P, Sharma P, Pant HC (2001). Cyclin-dependent protein kinase 5 (CDK5) and the regulation of neurofilament metabolism. Eur J Biochem 268: 1534-1546.

Greengard P (2001). The neurobiology of slow synaptic transmission. Science 294: 1024-1030.

Greengard P, Allen P, Nairn AC (1999). Beyond the dopamine receptor: the DARPP-32/protein phosphatase-1 cascade. Neuron 23: 435-437.

Groenewegen HJ, Mulder AB, Beijer AVJ, Wright CI, Lopes da Silva FH, Pennartz CMA (1999). Hippocampal and amygdaloid interactions in the nucleus accumbens. Psychobiology 27: 149-164.

Gupta A, Tsai LH (2001). A kinase to dampen the effects of cocaine. Science 292: 236-239.

Hsieh HC, Li HY, Lin MY, Chiou HY, Lin SY, Wong CH et al (2002). Differential expression of haloperidol-induced immediate early genes and CREB/ PCREB binding in the nucleus accumbens of amphetamine-sensitized rats. Synapse 45: 230-244.

Kalivas PW, Stewart J (1991). Dopamine transmission in the initiation and expression of drug- and stress-induced sensitization of motor activity. Brain Res Rev 16: 223-244.

Keiz M, Chen J, Carlezon WA, Whisler K, Gilden L, Beckmann AM et al (1999). Expression of the transcription factor $\Delta$ FosB in the brain controls sensitivity to cocaine. Nature 401: 272-275.

Koob GF (1996). Drug addiction: the yin and yang of hedonic homeostasis. Neuron 16: 893-896.

Kuczenski R, Segal DS (1999). Sensitization of amphetamineinduced stereotyped behaviors during the acute response. J Pharmacol Exp Ther 288: 699-709.

Lew J (1994). Neuronal cdc2-like kinase is a complex of cyclindependent kinase 5 and a novel brain-specific regulatory subunit. Nature 371: 423-425.

Liu F, Ma XH, Ule J, Bibb JA, Nishi A, Demaggio AJ et al (2001). Regulation of cyclin-dependent kinase 5 and casein 
kinase 1 by metabotropic glutamate receptors. Proc Natl Acad Sci 98: 11062-11068.

Lu L, Grimm JW, Shaham Y, Hope BT (2003). Molecular neuroadaptations in the accumbens and ventral tegmental area during the first 90 days of forced abstinence from cocaine selfadministration in rats. J Neurochem 85: 1604-1613.

Maccioni RB, Otth C, Concha II, Munoz JP (2001). The protein kinase cdk5. Eur J Biochem 268: 1518-1527.

Nestler EJ (2001). Molecular basis of long-term plasticity underlying addiction. Nat Rev 2: 119-128.

Nestler EJ, Aghajanian GK (1997). Molecular and cellular basis of addiction. Science 278: 58-63.

Nishi A, Bibb JA, Matsuyama S, Hamada M, Higashi H, Nairn AC et al (2002). Regulation of DARPP-32 dephosphorylation at PKA-and cdk5-sites by NMDA and AMPA receptors: distinct roles of calcineurin and protein phosphatase-2A. J Neurochem 81: 832-841.

Nishi A, Bibb JA, Snyder G, Higashi H, Nairn AC, Greengard P (2000). Amplification of dopaminergic signaling by a positive feedback loop. Proc Natl Acad Sci 97: 12840-12845.

Norrholm SD, Bibb JA, Nestler EJ, Ouimet CC, Taylor JR, Greengard P (2003). Cocaine-induced proliferation of dendritic spines in nucleus accumbens is dependent on the activity of cyclin-dependent kinase-5. Neuroscience 116: 19-22.

Paxinos G, Watson C (1996). The Rat Atlas in Stereotaxic Coordinates. Academic Press: San Diego.

Robinson TE, Becker JB (1986). Enduring changes in brain and behavior produced by chronic amphetamine administration: a review and evaluation of animal models of amphetamine psychosis. Brain Res Rev 11: 157-198.
Robinson TE, Kolb B (1997). Persistent structural modifications in nucleus accumbens and prefrontal cortex neurons produced by previous experience with amphetamine. J Neurosci 17: 8491-8497.

Robinson TE, Kolb B (1999). Alternations in the morphology of dendrites and dendritic spines in the nucleus accumbens and prefrontal cortex following repeated treatment with amphetamine or cocaine. Eur J Neurosci 11: 1598-1604.

Self DW, Nestler EJ (1995). Molecular mechanisms of drug reinforcement and addiction. Annu Rev Neurosci 18: 463-495.

Sharma P, Sharma M, Amin ND, Albers RW, Pant HC (1999). Regulation of cyclin-dependent kinase 5 catalytic activity by phorphorylation. Proc Natl Acad Sci USA 96: 11156-11160.

Smith DS, Greer PL, Tsai LH (2001). Cdk5 on the brain. Cell Growth Diff 12: 277-283.

Smith DS, Tsai LH (2002). Cdk5 behind the wheel: a role in trafficking and transport? Trends Cell Biol 12: 28-36.

Svenningsson P, Nishi A, Fisone G, Girault J-A, Nairn AC, Greengard P (2004). DARPP-32: an integrator of neurotransmission. Annu Rev Pharmacol Toxicol 44: 269-296.

Todtenkopf MS, Stellar JR (2000). Assessment of tyrosine hydroxylase immunoreactive innervation in five subregions of the nucleus accumbens shell in rats treated with repeated cocaine. Synapse 38: 261-270.

Vanderschuren LJMJ, Kalivas PW (2000). Alterations in dopaminergic and glutamatergic transmission in the induction and expression of behavioral sensitization: a critical review of preclinical studies. Psychopharmacology 151: 99-120.

White FJ, Cooper DC (2001). The vicious cyclin of addiction. Nat Med 7: 416-418. 